Focussed on "Crack Paths"

\title{
Microcrack interaction with circular inclusion and interfacial zone
}

\author{
Tomas Profant, Miroslav Hrstka \\ Brno University of Technology, Technická 2896/2, 61669 Brno, Czech Republic \\ profant@fme.vutbr.cz,mira.hrstka@gmail.com
}

Jan Klusák

CEITEC IPM, Institute of Physics of Materials; Zižkova 22, 616 62, Brno, Czech Republic

klusak@ipm.cz.

\begin{abstract}
A geometrically simplified plane elasticity problem of a finite small crack emanating from a thin interfacial zone surrounding the circular inclusion situated in the finite bounded domain is investigated. The crack is arbitrarily oriented and modelled using the distribution dislocation technique. This model represents the inner solution of the studied problem. The corresponding fundamental solution is based on the application of Muskhelishvili complex potentials in the form of the Laurent series. The coefficients of the series are evaluated from the compatibility conditions along the interfaces of the inclusion, the interfacial zone and the enclosing matrix. The fundamental solution is also used in the solution of the boundary integral method approximating the stress and strain relations of the so-called outer solution. The asymptotic analysis at the point of the crack initiation combines the inner and the outer solution and results in the evaluation of the stress intensity factors of the crack tip, which lies in the matrix. The topological derivative is subsequently used to approximate the energy release rate field associated with the perturbing crack in the matrix. The extreme values of the energy release rate allow one to assess the crack path direction of the initiated microcrack.
\end{abstract}

KEYwORDS. Crack path assessment; Complex potentials; Interfacial zone; Circular inclusion; Fundamental solution; Topological derivative.

\section{OPEN ACCESS}

Citation: Profant, T., Hrstka, M., Klusák, J., Microcrack interaction with circular inclusion and interfacial zone, Frattura ed Integrità Strutturale, 48 (2019) 503-512.

Received: 29.11.2018

Accepted: 22.01.2019

Published: 04.01.2019

Copyright: (C) 2019 This is an open access article under the terms of the CC-BY 4.0, which permits unrestricted use, distribution, and reproduction in any medium, provided the original author and source are credited.

\section{INTRODUCTION}

icrocracking is a phenomenon appearing in the vicinity of some stress rising features, where relatively short cracks rise and grow. An inclusion whose material properties differ from the ones of the surrounding matrix can be taken into account as a potential generator of such stresses [1], [2], [3]. The stress distribution generated by the presence of the inclusion can be influenced by its interfacial zone between the inclusion and the matrix. In spite of 
the idealization of the problem geometry, the analytical mathematical tools can propose an energetic balance at the crack tip initiated in this stress field. The energy consideration is a basic concept widely used not only in the fracture mechanics. The energy release rate (ERR) associated with a finite small and arbitrary oriented crack initiation at the interface between the interfacial zone of the circular inclusion and the matrix is considered to assess the direction of the crack path influenced by the presence of the inclusion and its interfacial zone. The topological derivative field indicates the variation of a response functional when an infinitesimal hole is introduced into the body, where the response functional is the total potential energy of the fractured matrix. As the first, it is introduced the fundamental solution in the form of the unit point force or edge dislocation interacting with the inclusion and the interfacial zone, [4]. The fundamental solution is found in the form of the Laurent series whose coefficients are evaluated from the compatibility conditions between the inclusion, interfacial zone and infinity matrix. The fundamental solution is applied to the boundary integral method and the continuously distributed dislocation method [5]. Under the assumption of a relatively short crack with respect to the inclusion and matrix dimensions, the asymptotic analysis [6] introduces the outer and inner solution of the problem represented by the uncracked finite matrix and cracked infinity matrix, respectively. The asymptotic analysis also demonstrates that the mismatch between the stress values along the outer boundary of the uncracked matrix and the stresses at the same points of the unbounded cracked matrix is proportional to the crack length. The energy momentum tensor [7], [8] and the approximation of the energy release rate for any crack size and orientation by means of a topological derivative can be evaluated from the inner solution and the corresponding stress intensity factors at the crack tip lying in the matrix.

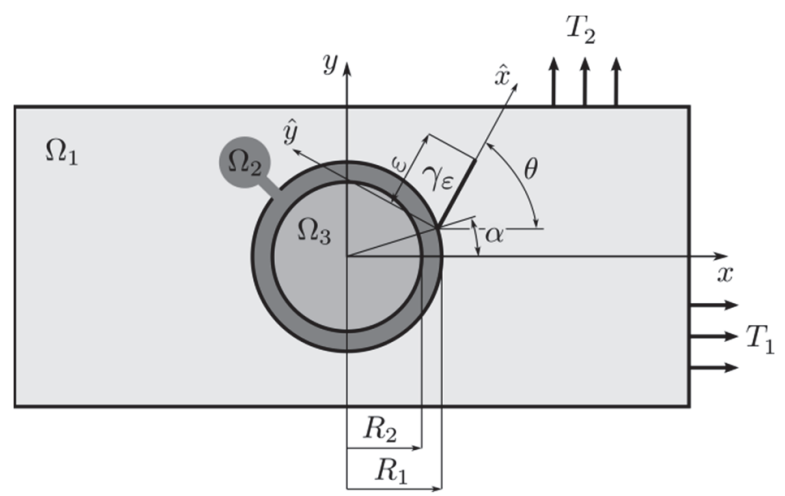

Figure 1: The problem formulation. The cracked matrix under the statically equilibrated external load and containing the inclusion with thin zone. The length of the crack is small with respect to the dimension of the inclusion.

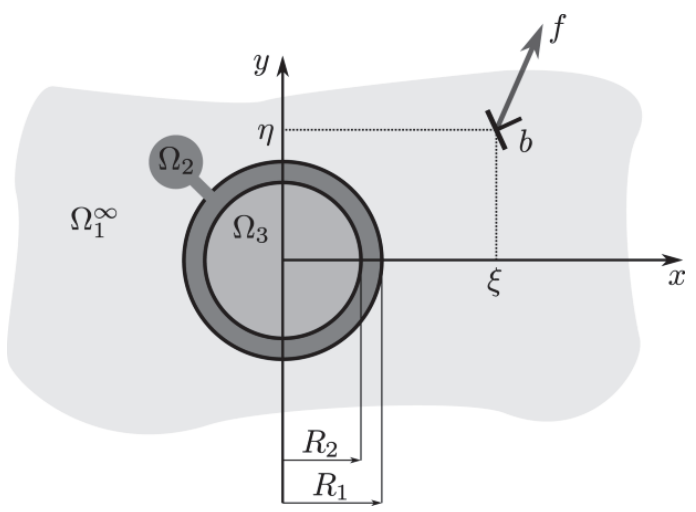

Figure 2: The point force $f$ and the dislocation with Burgers vector $b$ at the circular inclusion with the interfacial zone.

\section{FUNDAMENTAL SOLUTION}

7 he derivation of the fundamental solution, see Fig. 2, describing the interaction between the unit point force $f$ or dislocation with Burgers vector $b$ and the circular inclusion with an interfacial zone is based on [4], but contrary to its results it is supplemented by a rigid displacement of the body, because the fundamental solution is also to be 
applied in the method of the boundary integral equations as the outer solution of the problem. The basis for the expression of the fundamental solution is the complex analysis in the plane elasticity represented by Muskhelishvili's complex potentials, e.g. [10], which for the matrix $\Omega_{1}$ can be written in the form (the series of complex potential for domains $\Omega_{2}$ and $\Omega_{3}$ can be found in [3], [4])

$$
\begin{aligned}
& \phi_{1}(z ; \zeta)=\phi^{s}(z ; \zeta)+\sum_{n=0}^{\infty} h_{-n}(k, \gamma ; \zeta) z^{-n}=-\gamma \ln (z-\zeta)+\gamma \sum_{n=0}^{\infty} h_{-n}(k, \gamma ; \zeta) z^{-n}, \\
& \psi_{1}(z ; \zeta)=\psi^{s}(z ; \zeta)+\sum_{n=0}^{\infty} p_{-n}(k, \gamma ; \zeta) z^{-n}=-k \bar{\gamma} \ln (z-\zeta)+\gamma \frac{\bar{\zeta}}{z-\zeta}+\gamma \sum_{n=0}^{\infty} p_{-n}(k, \gamma ; \zeta) z^{-n},
\end{aligned}
$$

where

$$
\left\{\begin{array}{cc}
k=-\kappa_{1}, \gamma=\frac{f}{2 \pi\left(\kappa_{1}+1\right)} & \text { for point force, } \\
k=1, \gamma=\frac{\mathrm{i} \mu_{1} b}{\kappa_{1}+1} & \text { for dislocation. }
\end{array}\right.
$$

and $\mu_{1}$ is shear modulus and $\kappa_{1}$ is Kolosov's constant of the material of the domain $\Omega_{1}$. The functions $\phi^{s}(z ; \zeta)$ and $\psi^{s}(z ; \zeta)$ are the well-known complex potentials giving at the point $z=x+\mathrm{i} y$ the response of the point force $f=f_{x}+\mathrm{i} f_{y}$ or edge dislocation $b=b_{x}+\mathrm{i} b_{y}$ situated at point $\zeta=\xi+\mathrm{i} \eta$ in the infinite complex plane. The coefficients $h_{-n}(k, \gamma ; \zeta)$ and $p_{-n}(k, \gamma ; \zeta)$ are determined from the conditions of the continuity of displacements and the resultants of the tractions along the interfaces among the matrix $\Omega_{1}$, interfacial zone $\Omega_{2}$ and inclusion, see [3], [4].

\section{ASYMPTOTIC ANALYSIS AND STRESS INTENSITY FACTOR EVALUATION}

$\mathrm{T}$

he purpose of using the methods analyzing the path of the crack initiated from interface between the interfacial zone $\Omega_{2}$ and the matrix $\Omega_{1}$ follows from the stress composite expansion applied at the point of the crack initiation represented by the parameter $\alpha$, see Fig. 1. The composite expansion, i.e. an asymptotic expansion with respect to the small parameter $\varepsilon$, is introduced under the assumption that the crack $\gamma_{\varepsilon}$ is small with respect to the dimensions of the inclusion $\Omega_{3}$ and can be written as follows, see [3],

$$
\mathbf{T}(\mathbf{x}, \varepsilon)=\mathbf{T}^{(0)}(\mathbf{x})+\tilde{\mathbf{T}}^{(0)}(\mathbf{x} / \varepsilon)+\hat{\mathbf{T}}^{(0)}(\mathbf{x})
$$

where $\mathbf{x} \equiv[x, y], \mathbf{T}^{(0)}(\mathbf{x})$ is the outer stress, $\tilde{\mathbf{T}}^{(0)}(\mathbf{x} / \varepsilon)$ is the inner stress and $\hat{\mathbf{T}}^{(0)}(\mathbf{x})$ is an error when the members associated with $\varepsilon^{n}$, where $n>0$, are neglected in the asymptotic expansion of the stress $\mathbf{T}(\mathbf{x}, \varepsilon)$. The outer stress $\mathbf{T}^{(0)}(\mathbf{x})$ is defined in the crack-free domain $\Omega_{1}$ as the solution of the following boundary problem

$$
\begin{aligned}
& \nabla \cdot \mathbf{T}^{(0)}(\mathbf{x})=0 \text { for } \mathbf{x} \in \Omega \\
& \mathbf{T}^{(0)}(\mathbf{x}) \cdot \mathbf{n}=\mathbf{t}^{P} \text { for } \mathbf{x} \in \partial \Omega
\end{aligned}
$$

where

$$
\mathbf{t}^{P}= \begin{cases}\left(T_{1}, 0\right) & \text { for } \mathbf{n}=( \pm 1,0), \\ \left(0, T_{2}\right) & \text { for } \mathbf{n}=(0, \pm 1)\end{cases}
$$


and $\Omega=\Omega_{1} \cup \Omega_{2} \cup \Omega_{3}$ with outward normal $\mathbf{n}$. The boundary problem (4), (5) and (6) can only be solved using some numerical methods - the boundary element method in this case. This method ensures that the boundary conditions in ( 6 ) are fulfilled at discrete points along the boundary of the domain $\Omega$. On the other hand, the conditions of the stress and displacement compatibility at the interfaces of $\Omega_{1}, \Omega_{2}$ and $\Omega_{3}$ are satisfied automatically via the fundamental solution described in previous section.

The fundamental solution also makes it possible to express the boundary conditions for the inner problem represented in (3) by the stress tensor $\tilde{\mathbf{T}}^{(0)}(\mathbf{x} / \varepsilon)$, which must be independent of the crack length parameter $\varepsilon$. The inner problem can be formulated in such a way that a crack of the unit length $\left.\gamma_{1} \equiv \gamma_{\varepsilon}\right|_{\varepsilon=1}$ is initiated in arbitrary direction along the scaled $\hat{X}=\hat{x} / \varepsilon$-axis from the interface between the unloaded matrix $\Omega_{1}^{\infty}$ and the interfacial zone $\Omega_{2}$. The domain $\Omega_{1}^{\infty}$ is a domain $\Omega_{1}$ with an outer boundary extended to infinity. The crack faces are loaded with radial and tangential stresses $T_{\theta \theta}^{(0)}(\mathbf{x})$ and $T_{r \theta}^{(0)}(\mathbf{x})$ from the outer problem (4), (5) and (6), which are generated in the uncracked matrix $\Omega_{1}$ at points of the crack $\gamma_{\varepsilon}$. Thus,

$$
\begin{aligned}
& \nabla \cdot \tilde{\mathbf{T}}^{(0)}(\mathbf{X})=0 \text { for } \mathbf{X} \in \Omega_{\varepsilon}^{\infty} \\
& \tilde{\mathbf{t}}=\mathbf{T}^{(0)}(\varepsilon \mathbf{X}) \cdot \hat{\mathbf{n}} \text { for } \mathbf{X} \in \gamma_{1} \\
& \tilde{\mathbf{T}}^{(0)}(\mathbf{X}) \rightarrow 0 \text { for }|\mathbf{X}| \rightarrow \infty
\end{aligned}
$$

where $\Omega_{\varepsilon}^{\infty}$ is a cracked domain $\Omega^{\infty}=\Omega_{1}^{\infty} \cup \Omega_{2} \cup \Omega_{3}$. The inner problem is due to the scaled coordinate $\mathbf{X}=\mathbf{x} / \varepsilon$ independent of the parameter $\varepsilon$. Similarly to [9], the crack in (7), (8) and (9) is modelled using the continuously distributed dislocation technique [3], [5].

If the dislocation with Burgers vector $b=b_{x}+\mathrm{i} b_{y}$ is situated at the point $\mathrm{Z}=\zeta / \varepsilon \in \Omega_{1}^{\infty}$, then the stress tensor components $T_{Y Y}$ and $T_{X Y}$ appearing at the point $\mathscr{z}=z / \varepsilon \in \Omega_{1}^{\infty}$ can be written as follows using the potentials (1) and $(2)$

$$
\begin{aligned}
& T_{Y Y}(\mathscr{Z} ; \mathrm{Z})+\mathrm{i} T_{X Y}(\mathscr{Z} ; \mathrm{Z})=\frac{\mathrm{i} \mu_{1}}{\kappa_{1}+1}\left[-b \frac{1}{\mathscr{Z}-\mathrm{Z}}+b \frac{\overline{\mathscr{Z}}-\overline{\mathrm{Z}}}{(\mathscr{Z}-\mathrm{Z})^{2}}+2 \bar{b} \frac{1}{\mathscr{Z}-\mathrm{Z}}+\right. \\
& +\sum_{n=0}^{\infty} n\left[-b h_{-n}(k, \gamma ; \mathrm{Z}) \mathscr{Z}^{-n-1}+\overline{b h}_{-n}(k, \gamma ; \mathrm{Z}) \overline{\boldsymbol{Z}}^{-n-1}+\right. \\
& \left.\left.+b(n+1) b_{-n}(k, \gamma ; \mathrm{Z}) \overline{\mathscr{Z}} \mathscr{\sigma}^{-n-2}-b p_{-n}(k, \gamma ; \mathrm{Z}) \mathscr{Z}^{-n-1}\right]\right]
\end{aligned}
$$

The dislocations will be distributed along the $\hat{X}=\hat{x} / \varepsilon$-axis, i.e. for $\hat{Y}=\hat{y} / \varepsilon=0$, see Fig. 1. Hence the standard transformations of vectors and tensors with respect to the rotations through angles $\alpha$ and $\theta$ in $X Y$ and $\hat{X} \hat{Y}$ planes, respectively, must be applied to the Eqn. (10), see [5]. The singular part represented by the first three expressions in (10) is simply transformed to the Cauchy type singular kernel. The transformed regular part of (10) represented by the infinite sum can be written as

$$
\begin{aligned}
H_{\hat{X} \hat{Y}}(\hat{X} ; \hat{\Xi})= & H_{X X X}(\mathbf{X} ; \boldsymbol{\Xi}) \cos \theta \sin ^{2} \theta+H_{X Y Y}(\mathbf{X} ; \boldsymbol{\Xi}) \cos ^{3} \theta-H_{X X Y}(\mathbf{X} ; \boldsymbol{\Xi}) \cos \theta \sin 2 \theta+ \\
& +H_{Y X X}(\mathbf{X} ; \boldsymbol{\Xi}) \sin ^{3} \theta+H_{Y Y Y}(\mathbf{X} ; \boldsymbol{\Xi}) \cos ^{2} \theta \sin \theta-H_{Y X Y}(\mathbf{X} ; \boldsymbol{\Xi}) \sin \theta \sin 2 \theta \\
H_{\hat{Y} \hat{Y} \hat{Y}}(\hat{X} ; \hat{\Xi})= & -H_{X X X}(\mathbf{X} ; \boldsymbol{\Xi}) \sin ^{3} \theta-H_{X Y Y}(\mathbf{X} ; \boldsymbol{\Xi}) \cos ^{2} \theta \sin \theta+H_{X X Y}(\mathbf{X} ; \boldsymbol{\Xi}) \sin \theta \sin 2 \theta+ \\
& +H_{Y X X}(\mathbf{X} ; \boldsymbol{\Xi}) \cos \theta \sin ^{2} \theta+H_{Y Y Y}(\mathbf{X} ; \boldsymbol{\Xi}) \cos ^{3} \theta-H_{Y X Y}(\mathbf{X} ; \boldsymbol{\Xi}) \cos \theta \sin 2 \theta
\end{aligned}
$$




$$
\begin{aligned}
H_{\hat{X} \hat{X} \hat{Y}}(\hat{X} ; \hat{\Xi})= & -H_{X X X}(\mathbf{X} ; \boldsymbol{\Xi}) \cos ^{2} \theta \sin \theta+H_{X Y Y}(\mathbf{X} ; \boldsymbol{\Xi}) \cos ^{2} \theta \sin \theta-H_{X X Y}(\mathbf{X} ; \boldsymbol{\Xi}) \cos \theta \cos 2 \theta- \\
& -H_{Y X X}(\mathbf{X} ; \boldsymbol{\Xi}) \cos \theta \sin ^{2} \theta+H_{Y Y Y}(\mathbf{X} ; \boldsymbol{\Xi}) \cos \theta \sin ^{2} \theta-H_{Y X Y}(\mathbf{X} ; \boldsymbol{\Xi}) \cos 2 \theta \sin \theta, \\
H_{\hat{Y} \hat{X} \hat{Y}}(\hat{X} ; \hat{\Xi})= & H_{X X X}(\mathbf{X} ; \boldsymbol{\Xi}) \cos \theta \sin ^{2} \theta-H_{X Y Y}(\mathbf{X} ; \boldsymbol{\Xi}) \cos \theta \sin ^{2} \theta-H_{X X Y}(\mathbf{X} ; \boldsymbol{\Xi}) \cos 2 \theta \sin \theta+ \\
& -H_{Y X X}(\mathbf{X} ; \boldsymbol{\Xi}) \cos ^{2} \theta \sin \theta+H_{Y Y Y}(\mathbf{X} ; \boldsymbol{\Xi}) \cos ^{2} \theta \sin \theta-H_{Y X Y}(\mathbf{X} ; \boldsymbol{\Xi}) \cos \theta \cos 2 \theta,
\end{aligned}
$$

where

$$
\mathbf{X}=\left[R_{1} \cos \alpha+\hat{X} \cos \theta, R_{1} \sin \alpha+\hat{X} \sin \theta\right], \quad \boldsymbol{\Xi}=\left[R_{1} \cos \alpha+\hat{\Xi} \cos \theta, R_{1} \sin \alpha+\hat{\Xi} \sin \theta\right]
$$

and

$$
\begin{aligned}
& H_{X X X}(\mathbf{X} ; \boldsymbol{\Xi})=\sum_{n=1}^{\infty} n \Im\left\{h_{-n}(k, \gamma ; \mathrm{Z}) \mathscr{Z}^{-n-1}-\bar{b}_{-n}(k, \gamma ; \mathrm{Z}) \overline{\mathscr{J}}^{-n-1}+\right. \\
& \left.+(n+1) h_{-n}(k, \gamma ; \mathrm{Z}) \overline{\mathscr{Z}} \mathscr{W}^{-n-2}-p_{-n}(k, \gamma ; \mathrm{Z}) \mathscr{Z}^{-n-1}\right\}, \\
& H_{X Y Y}(\mathbf{X} ; \boldsymbol{\Xi})=\sum_{n=1}^{\infty} n \Im\left\{h_{-n}(k, \gamma ; Z) \mathscr{Z}^{-n-1}-\bar{h}_{-n}(k, \gamma ; Z) \overline{\mathscr{Z}}^{-n-1} p-\right. \\
& \left.-(n+1) h_{-n}(k, \gamma ; \mathrm{Z}) \overline{\mathscr{Z}} \mathscr{Z}^{-n-2}+p_{-n}(k, \gamma ; \mathrm{Z}) \mathscr{Z}^{-n-1}\right\}, \\
& H_{X X Y}(\mathbf{X} ; \boldsymbol{\Xi})=\sum_{n=1}^{\infty} n \Re\left\{-h_{-n}(k, \gamma ; Z) \mathscr{Z}^{-n-1}+\bar{b}_{-n}(k, \gamma ; \mathrm{Z}) \overline{\boldsymbol{Z}}^{-n-1}+\right. \\
& \left.+(n+1) b_{-n}(k, \gamma ; \mathrm{Z}) \overline{\mathscr{\sigma}} \mathscr{\mho}^{-n-2}-p_{-n}(k, \gamma ; \mathrm{Z}) \mathscr{Z}^{-n-1}\right\}, \\
& H_{Y X X}(\mathbf{X} ; \boldsymbol{\Xi})=\sum_{n=1}^{\infty} n \Re\left\{h_{-n}(k, \gamma ; \mathbf{Z}) \mathbb{Z}^{-n-1}+\bar{b}_{-n}(k, \gamma ; \mathbf{Z}) \overline{\mathcal{Z}}^{-n-1}+\right. \\
& \left.+(n+1) b_{-n}(k, \gamma ; \mathrm{Z}) \overline{\mathscr{L}} \mathscr{Z}^{-n-2}-p_{-n}(k, \gamma ; \mathrm{Z}) \mathscr{Z}^{-n-1}\right\}, \\
& H_{Y Y Y}(\mathbf{X} ; \boldsymbol{\Xi})=\sum_{n=1}^{\infty} n \Re\left\{h_{-n}(k, \gamma ; Z) \mathscr{Z}^{-n-1}+\bar{b}_{-n}(k, \gamma ; Z) \overline{\mathscr{J}}^{-n-1}-\right. \\
& \left.-(n+1) b_{-n}(k, \gamma ; \mathrm{Z}) \overline{\mathscr{L}} \mathscr{Z}^{-n-2}+p_{-n}(k, \gamma ; \mathrm{Z}) \mathscr{Z}^{-n-1}\right\}, \\
& H_{Y X Y}(\mathbf{X} ; \boldsymbol{\Xi})=\sum_{n=1}^{\infty} n \Im\left\{h_{-n}(k, \gamma ; Z) \mathscr{Z}^{-n-1}+\bar{b}_{-n}(k, \gamma ; Z) \overline{\mathscr{Z}}^{-n-1}-\right. \\
& \left.-(n+1) h_{-n}(k, \gamma ; \mathrm{Z}) \overline{\mathscr{Z}} \mathscr{Z}^{-n-2}+p_{-n}(k, \gamma ; \mathrm{Z}) \mathscr{Z}^{-n-1}\right\} .
\end{aligned}
$$

The symbols $\Re\{$.$\} and \Im\{$.$\} mean real and imaginary value of the complex expression. The dislocation is continuously$ distributed along the $\hat{x} / \varepsilon$-axis in the interval $\hat{\Xi} \in(0,1)$. This process is modelled by introducing the density of the Burgers vector $B_{\hat{Y}}(\hat{\Xi})$ and $B_{\hat{X}}(\hat{\Xi})$, which is in relation with Burgers vector $\mathbf{b}=\left(b_{\hat{X}}, b_{\hat{Y}}\right)$ as follows

$$
B_{\hat{Y}}(\hat{\Xi})=\frac{\mathrm{d} b_{\hat{Y}}(\hat{\Xi})}{\mathrm{d} \hat{\Xi}}, \quad B_{\hat{X}}(\hat{\Xi})=\frac{\mathrm{d} b_{\hat{X}}(\hat{\Xi})}{\mathrm{d} \hat{\Xi}},
$$


where

$$
\begin{aligned}
& b_{\hat{X}}=b_{X} \cos \theta+b_{Y} \sin \theta, \\
& b_{\hat{Y}}=-b_{X} \sin \theta+b_{Y} \cos \theta .
\end{aligned}
$$

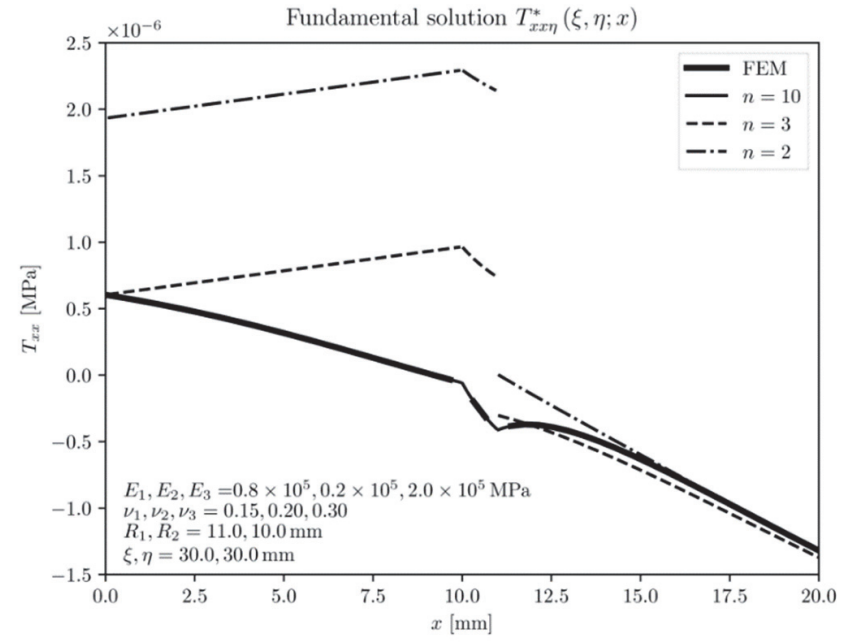

Figure 3: The stress tensor component $T_{x x}$ of the fundamental solution $\mathbf{T}^{*}(\xi, \eta ; x)$ compared with the FEM solution along the positive $x$ - axis for the unit point force $f=f_{\eta}=1$ situated at the point $[\xi, \eta]$.

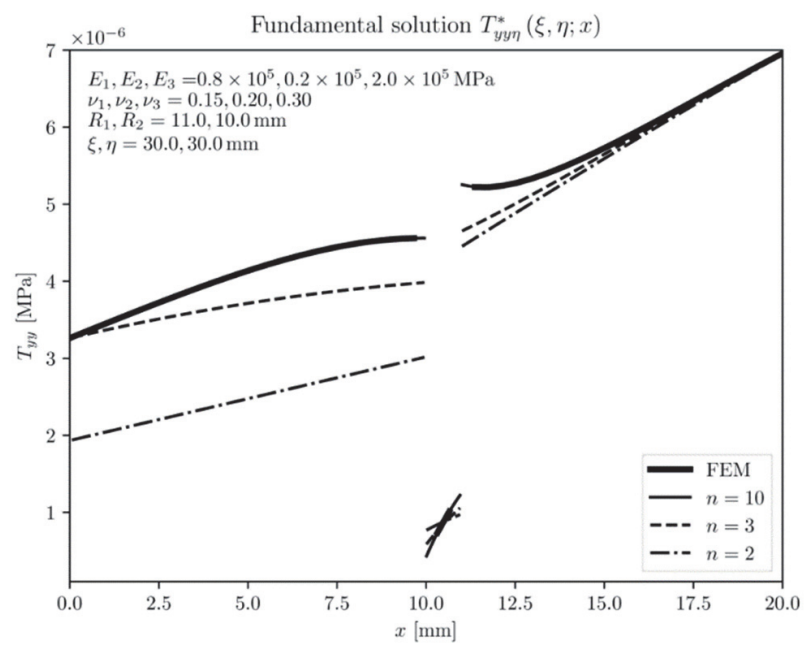

Figure 5: The stress tensor components $T_{y y}$ of the fundamental solution $\mathbf{T}^{*}(\xi, \eta ; x)$ compared with the FEM solution along the positive $x$-axis for the unit point force $f=f_{\eta}=1$ situated at the point $[\xi, \eta]$.

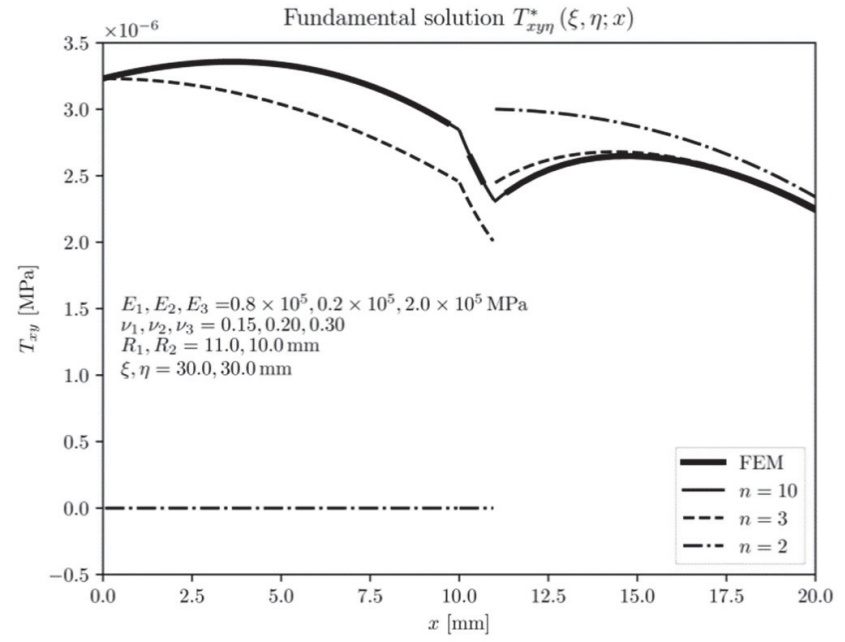

Figure 4: The stress tensor components $T_{x y}$ of the fundamental solution $\mathbf{T}^{*}(\xi, \eta ; x)$ compared with the FEM solution along the positive $x$-axis for the unit point force $f=f_{\eta}=1$ situated at the point $[\xi, \eta]$.

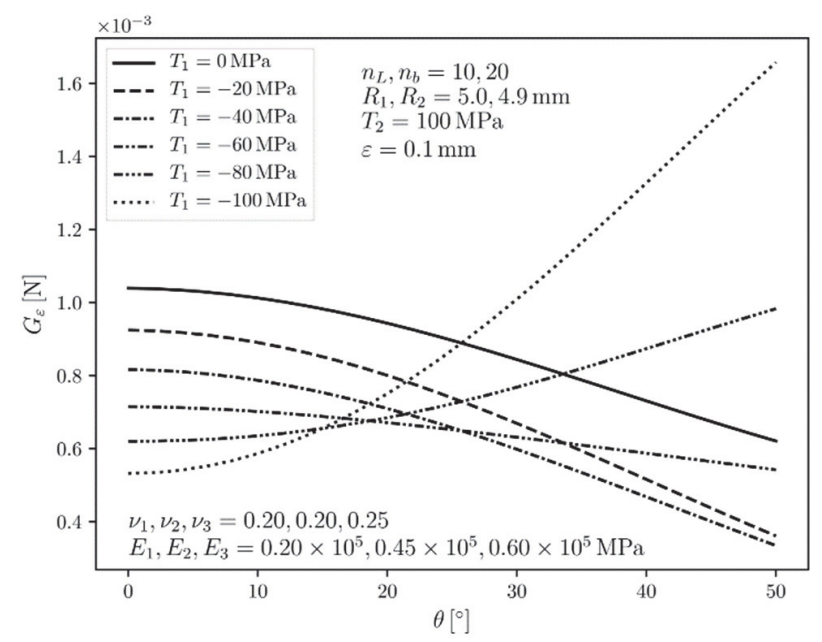

Figure 6: The energy release rate $G_{\varepsilon}$ as a function of the crack inclination $\theta$ and the outer stress $T_{1}$ when $\alpha=0^{\circ}$. 


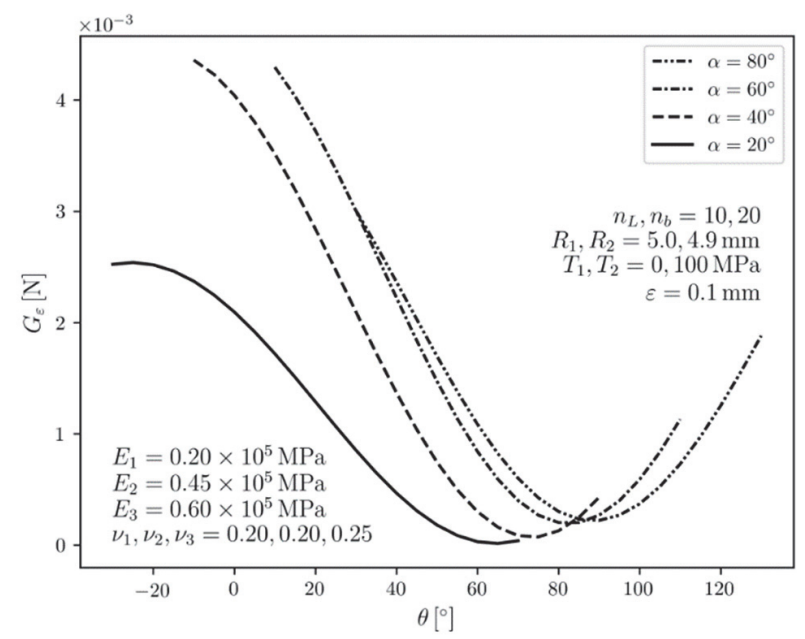

Figure 7: The energy release rate $G_{\varepsilon}$ as a function of the crack inclination $\theta$ and the crack initiation angle $\alpha$.

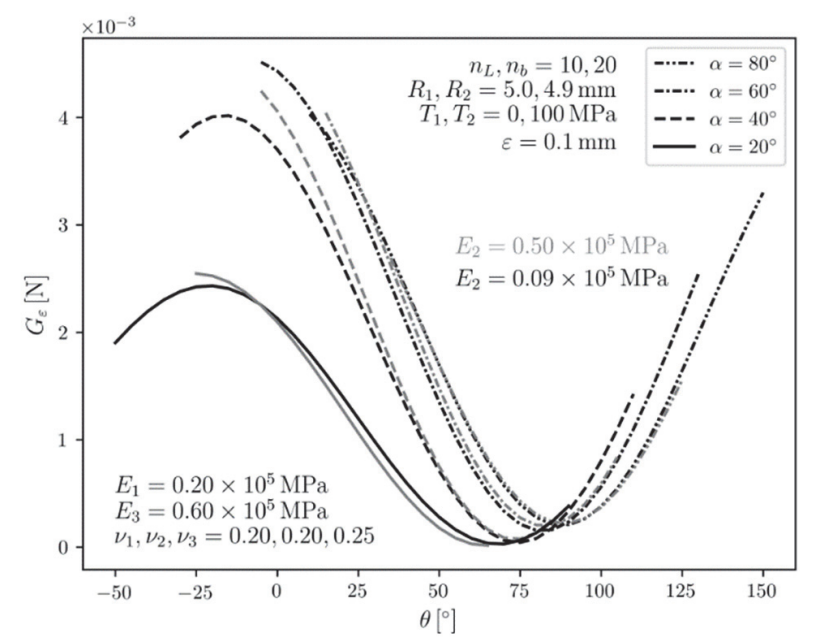

Figure 8: The energy release rate $G_{\varepsilon}$ as a function of the crack inclination $\theta$, the crack initiation angle $\alpha$ and elastic modulus of the interfacial zone $E_{2}$.

Finally, the boundary conditions (8) and (9) in the inner problem formulation (7), lead to the solution of the following system of the singular integral equations following from the above mentioned transformations of (10)

$$
\begin{aligned}
& T_{\hat{y} \hat{y}}^{(0)}(\varepsilon \hat{X})=\frac{\mu_{1}}{\pi\left(\kappa_{1}+1\right)} \int_{0}^{1}\left[B_{\hat{X}}(\hat{\Xi}) H_{\hat{X} \hat{Y} \hat{Y}}(\hat{X} ; \hat{\Xi})+B_{\hat{Y}}(\hat{\Xi})\left(\frac{2}{\hat{X}-\hat{\Xi}}+H_{\hat{Y} \hat{Y} \hat{Y}}(\hat{X} ; \hat{\Xi})\right)\right] \mathrm{d} \hat{\Xi}, \\
& T_{\hat{x} \hat{y}}^{(0)}(\varepsilon \hat{X})=\frac{\mu_{1}}{\pi\left(\kappa_{1}+1\right)} \int_{0}^{1}\left[B_{\hat{X}}(\hat{\Xi})\left(\frac{2}{\hat{X}-\hat{\Xi}}+H_{\hat{X} \hat{X} \hat{Y}}(\hat{X} ; \hat{\Xi})\right)+B_{\hat{Y}}(\hat{\Xi}) H_{\hat{Y} \hat{X} \hat{Y}}(\hat{X} ; \hat{\Xi})\right] \mathrm{d} \hat{\Xi}
\end{aligned}
$$

and integral equations ensuring the closed crack tips

$$
\int_{0}^{1} B_{\hat{X}}(\hat{\Xi}) \mathrm{d} \hat{\Xi}=0, \quad \int_{0}^{1} B_{\hat{Y}}(\hat{\Xi}) \mathrm{d} \hat{\Xi}=0
$$

The outer stresses $T_{\hat{y} y}^{(0)}(\hat{x}), T_{\hat{x} \hat{y}}^{(0)}(\hat{x})$ are the solution of (4), (5) and (6) at the points $\hat{x}=\varepsilon \hat{X}$ of the crack $\gamma_{\varepsilon}$ in the case of the loaded but uncracked matrix $\Omega_{1}$ and also linearly transformed with respect to the local coordinate system $\hat{x y}$. The unknown Burgers vector densities $B_{\hat{X}}(\hat{\Xi})$ and $B_{\hat{Y}}(\hat{\Xi})$ are found in the form

$$
B_{\hat{Y}}(\hat{\Xi})=\hat{\Xi}^{\lambda}(1-\hat{\Xi})^{-\frac{1}{2}} \chi_{\hat{Y}}(\hat{\Xi}), \quad B_{\hat{X}}(\hat{\Xi})=\Xi^{\lambda}(1-\hat{\Xi})^{-\frac{1}{2}} \chi_{\hat{X}}(\hat{\Xi}),
$$

where the value of the exponent $\lambda$ is different from the value -0.5 due to the mismatch of the materials of the matrix $\Omega_{1}$ and interfacial zone $\Omega_{2}$. The functions $\chi_{\hat{Y}}(\hat{\Xi})$ and $\chi_{\hat{X}}(\hat{\Xi})$ are continuous in the interval $\hat{\Xi} \in(0,1)$ and approximated by Jacobi polynomials. The stress intensity factors $K_{I}$ and $K_{I I}$ at the crack tip lying in the matrix $\Omega_{1}$ can be evaluated from the dislocation densities $B_{\hat{Y}}(\hat{\Xi})$ and $B_{\hat{X}}(\hat{\Xi})$ as follows

$$
K_{I, I I}=2^{\frac{1}{2}+\lambda} \sqrt{\pi} \frac{\mu_{1}}{\kappa_{1}+1} \chi_{\hat{Y}, \hat{X}}(1)
$$




\section{ENERGY RELEASE RATE EVALUATION}

$\mathrm{F}$

or the purpose of the assessment of the crack path direction, the knowledge of the stress intensity factors at the tips of the just existing crack is insufficient and the change of the energy state of the unit crack advance is necessary. The shape optimization methods tender an interesting way to eliminate the problem of the complexity of the geometry of the presented problem. The shape functional $U\left(\Omega_{\varepsilon}\right)$ plays an important role in the following method, because represents the potential energy of the fracture problem (3). The change of the shape functional with respect to the small perturbation $\delta \varepsilon$ of the crack $\gamma_{\varepsilon}$ at its tip $\hat{x}=\varepsilon$ can be expressed by its asymptotic expansion, see [3], [8] and [9],

$$
U\left(\Omega_{\varepsilon+\delta \varepsilon}\right)=U\left(\Omega_{\varepsilon}\right)+\left.g(\delta \varepsilon) J(\hat{x})\right|_{\hat{x}=\varepsilon}+o(\delta \varepsilon),
$$

where $J$ is the topological derivative of $U$ on the perturbed domain $\Omega_{\varepsilon+\delta \varepsilon}$. It is supposed that $g(\varepsilon)$ is the positive function such that $g(\delta \varepsilon) \rightarrow 0$ with $\delta \varepsilon \rightarrow 0$. The additional requirements about the remainder of (29) can be found in [8]. In [8] it is also shown the relation between the shape derivative of $U\left(\Omega_{\varepsilon}\right)$ and the topological one in the sense to the domain variation produced by an uniform expansion of some perturbation. This method, the so-called topological-shape sensitivity method, uses the concept of shape sensitivity analysis as an intermediate step in the topological derivative calculation. From the combination of the topological and shape sensitivity one gets the expression of the energy release rate, [3], [9],

$$
\left.G_{\varepsilon}(\hat{x})\right|_{\hat{x}=\varepsilon}=\left.\dot{J}(\hat{x})\right|_{\hat{x}=\varepsilon}=-\lim _{\delta \varepsilon \rightarrow 0} \int_{\partial B_{\delta \varepsilon}}\left(\Sigma_{\varepsilon} \cdot \mathbf{n}\right) \cdot \mathbf{e}_{\hat{x}} \mathrm{~d} S=\frac{\varepsilon}{\bar{E}}\left[K_{I}^{2}+K_{I I}^{2}\right]
$$

where $\bar{E}=E_{1} /\left(1-\nu_{1}\right)$ for plane strain, $\bar{E}=E_{1}$ for plane stress, $\dot{J}(\hat{x})$ is a derivative of $J(\hat{x})$ with respect to $\delta \varepsilon, \Sigma_{\varepsilon}$ is the Eshelby's momentum tensor [7], $\mathbf{e}_{\hat{x}}$ is a basis vector of $\hat{x}$-axis and $\mathbf{n}$ is an unit normal to the boundary $\partial B_{\delta \varepsilon}$ of the stress-free circular shaped perturbation $B_{\delta \varepsilon}$ at the crack tip $\hat{x}=\varepsilon$.

\section{NUMERICAL RESULTS}

A correct description of the stress field surrounding the inclusion $\Omega_{3}$ with the interfacial zone $\Omega_{2}$ situated in the matrix $\Omega_{1}$ is conditioned by the fundamental solution (1) behavior at the inclusion, interfacial zone and matrix interfaces. The Figs. 3-5 show a numerical example of the convergence and the fulfilling of the compatibility conditions of the stress component $T_{x x}, T_{x y}$ and $T_{y y}$ of the stress tensor $\mathbf{T}^{*}(\xi, \eta ; \mathbf{x})$ along the positive $x$-axis ( $\mathbf{x}=[x, 0], x>0)$ generated by the fundamental solution (1) for the domain $\Omega_{1}$ and its additional forms for the domain $\Omega_{2}$ and $\Omega_{3}$ along their interfaces. The applied values of elastic moduli, geometry and degree of Laurent series are also added. The unit point force $f$ is parallel with respect to $y \equiv \eta$-axis and situated at a point $[\xi, \eta]$. The graphs show the matching of the Laurent approximation of the fundamental solution and the FEM solution along the positive $x$-axis for series degree $n=10$ (especially in the interval $x \in(10,11) \mathrm{mm}$, where the interfacial zone is situated).

In the next numerical example, see Fig. 6 , the case of the assessment of the crack path direction $\theta_{0}$ of the crack initiated from the point on the interface between the matrix and interfacial zone corresponding to $\alpha=0^{\circ}$ is considered. The elastic moduli of the used materials $\left(E_{1}, \nu_{1}\right),\left(E_{2}, \nu_{2}\right),\left(E_{3}, \nu_{3}\right)$, the finite crack size $\varepsilon$, geometry $R_{1}, R_{2}$ and degree of Laurent series are also given in the graph. The graph shows the dependence of the corresponding energy release rate $G_{\varepsilon}$ on $\theta$, where the external load $T_{1}$ varies from zero value to the negative value of $T_{2}$ to model the shear loading of the cracked matrix. The crack initiation direction corresponding to the local maximum value of $G_{\varepsilon}$ is of course fairly 
influenced by the sheer stresses and mode II loading. It is worth mentioning that the maximum value of the angle $\theta \sim 50^{\circ}$ is due to the value of the stress singularity exponent $\lambda$, which becomes complex for the $\theta>50^{\circ}$. The complex value of $\lambda$ requires different approach of the stress field description. The graph also shows that for some combination of the external stresses $T_{1}$ getting some specific value from the range $T_{1} \in(-80,-60) \mathrm{MPa}$ it is difficult to assess the crack path direction, because the dependence of the energy release rate $G_{\varepsilon}$ is rather flat without the clear local extremal value.

The Fig. 7 shows the position of the crack initiation at the interface between the interfacial zone and matrix represented by the angle $\alpha$ and the crack inclination $\theta$. The elastic moduli of the used materials $\left(E_{1}, \nu_{1}\right),\left(E_{2}, \nu_{2}\right),\left(E_{3}, \nu_{3}\right)$, the finite crack size $\varepsilon$, geometry $R_{1}, R_{2}$ and degree of Laurent series are again given in the graph. The external load $T_{1}$ is zero in this case, while $T_{2}=100 \mathrm{MPa}$. The local maximum value of $G_{\varepsilon}$ is for $\alpha \sim 40^{\circ}$ and the crack path direction $\theta_{0}$ is near the zero value, i.e. the crack growth under the mode I loading. Similarly to the previous example, the maximum values of $G_{\varepsilon}$ are limited due to the real values of the stress singularity exponent $\lambda$.

The influence of the interfacial zone on the crack initiation position and potentially crack growing direction shows Fig. 8. The width of the interfacial zone $\Omega_{2}$ plays an unimportant role in the crack behavior and it is not illustrated there. On the other hand, the material properties of $\Omega_{2}$ influence the point of the crack initiation as well as the crack path direction. The grey curves represent the material of the interfacial zone with elastic moduli similar to those of the stiffer particle $\Omega_{3}$, the black ones similar to the material softer then the matrix $\Omega_{1}$. On can see that the position of the point of the crack initiation represented by the angle $\alpha$ tends to the lower values for the stiffer material of $\Omega_{2}$ and the crack path direction $\theta_{0}$ moves to the positive values.

\section{CONCLUSIONS}

1 he energy release rate associated with the arbitrary oriented finite small crack initiating at the interface between the interfacial zone of the circular inclusion and the matrix was evaluated. The scheme of the energy release rate evaluation was adopted from [9] but modified to the presented problem. The proposed procedure combines traditional as well as modern analytical approaches with a numerical one. The most important part of the analysis is the establishing of the fundamental solution given by the interaction between the edge dislocation or point force and circular inclusion with an interfacial zone. A numerical example of the convergence of the approximating series of the fundamental solution was shown. The asymptotic analysis, where the crack perturbs the matrix only and the crack has to have a finite small length, allows one to apply the fundamental solution to model as the cracked infinity as the uncrack finite loaded matrix containing the inclusion. The numerical results showed the influence of the external loads on the crack path direction but also the position of its point of initiation along the interface between the interfacial zone and matrix. The influence of the material properties of the interfacial zone on the cracking of the matrix was also shown. Even that the character of the presented results is theoretical only and their validation is desirable, the applied procedure offers an interesting tools to solve the fracture problems of the composites with sound mathematical background.

\section{ACKNOWLEDGMENTS}

7 his research has been financially supported by the Czech Science Foundation through the Grant 16-18702S and by the Ministry of Education, Youth and Sports of the Czech Republic under the project CEITEC 2020 [LQ1601].

\section{REFERENCES}

[1] Krepl, O., Klusák, J. (2017). The influence of non-singular terms on the precision of stress description near a sharp material inclusion tip, Theor. Appl. Fract. Mech., 90, pp. 85-99, DOI: 10.1016/J.TAFMEC.2017.03.007.

[2] Krepl, O., Klusák, J. (2016). Reconstruction of a 2D stress field around the tip of a sharp material inclusion, Procedia Struct. Integr., 2, pp. 1920-7, DOI: 10.1016/J.PROSTR.2016.06.241. 
[3] Profant, T., Hrstka, M., Klusák, J. (2019). An asymptotic analysis of crack initiation from an interfacial zone surrounding the circular inclusion, Compos. Struct., 208, pp. 479-97, DOI: 10.1016/J.COMPSTRUCT.2018.10.020.

[4] Cheeseman, B.A., Santare, M.H. (2001). The effect of the interphase on crack-inclusion interactions, Int. J. Fract., 109(3), pp. 303-23, DOI: 10.1023/A:1011092801013.

[5] Hills, D., Kelly, P., Dai, D., Korsunsky, A. (1999). Solution of Crack Problems: The Distributed Dislocation Technique, Amsterdam, Kluwer Academic Publishers.

[6] Kozlov, V., Maz’ya, V., Movchan, A. (1999). Asymptotic Analysis of Fields in Multi-structures, Oxford, Oxford University Press.

[7] Eshelby, J.D. (1975). The elastic energy-momentum tensor, J. Elast., 5(3-4), pp. 321-35, DOI: $10.1007 /$ BF00126994.

[8] Novotny, A.A., Sokołowski, J. (2013). Topological Derivatives in Shape Optimization, Springer-Verlag Berlin Heidelberg.

[9] Silva, M., Geubelle, P.H., Tortorelli, D.A. (2011). Energy release rate approximation for small surface-breaking cracks using the topological derivative, J. Mech. Phys. Solids, 59(5), pp. 925-39, DOI: 10.1016/J.JMPS.2011.03.005.

[10] Muskhelishvili, N.I. (1977). Some Basic Problems of the Mathematical Theory of Elasticity, Springer Netherlands. 